\title{
XLVIII. The photoelectric properties of thin metal films
}

\section{J. Robinson M.Sc. Ph.D.}

To cite this article: J. Robinson M.Sc. Ph.D. (1912) XLVIII. The photoelectric properties of thin metal films, Philosophical Magazine Series 6, 23:136, 542-551, DOI:

10.1080/14786440408637247

To link to this article: http://dx.doi.org/10.1080/14786440408637247

曲 Published online: 20 Apr 2009.

Submit your article to this journal \lceil

Џ Article views: 2

Q View related articles $\square$

Citing articles: 7 View citing articles 5 
All observations of complete radiation show that $H_{m}$ falls off when $h$ is large, that is when the temperature is small, in some such ratio as $e^{-\alpha_{h}}$ where $a$ is inversely as the wavelength. Hence

$$
\int_{h}^{\infty} \bar{H}_{m} d h
$$

is finite and by integration of (101) it follows that $\psi(h)$ bears a finite ratio to $\psi(\infty)$ where

$$
\psi(h)=\int_{0}^{\infty} e^{-h H_{m}} f\left(H_{m}\right) d H_{m}
$$

This, it can easily be seen, is impossible if $f\left(H_{m}\right)$ is not somewhere infinite. Planck has in fact, while retaining the formula (101), supposed that $f\left(H_{m}\right)$ is zero except at a set of points distributed at equal intervals where it becomes infinite.

\section{Conclusion.}

It now appears that with no continuous laws of motion is it possible to account for the actual distribution of energy. The proof, however, involves the assumption that the statistical method applies to the æther with its infinity of degrees of freedom. Since this paper was completed I have succeeded in extending the direct calculation of emission and absorption to matter obeying any continuous laws of motion whatever. That I reserve for another paper ; in this the direct method has shown that for any material system equipartition follows if the principle of least action is assumed.

January 29, 1912.

XLVIII. The Photoelectric Properties of Thin Metal Films. By J. Robinson, M.Se., Ph.D., Demonstrator in Physics at the University of Sheffield *

7 THE photoelectric effect of thin metal films on quartz 1 has been investigated by Kleeman $\dagger$ and by Stuhlmann $\ddagger$, both of whom found a difference in the effects produced by incident and emergent lights. For thin films the emergent light produces a larger ionization current than incident light. Stuhlmann showed further that the reverse is the case for thick films. Both investigators worked with films in air at atmospheric pressure. Kleeman also made a measurement at a low pressure.

* Communicated by the Author.

† Kleeman, Proc. Roy. Soc. Ixxxiv. 1910, p. 93.

f O. Stuhlmann, Phil. Mag. xx. 1910, p. 331. 
It has been found that the photoelectric properties of metal films vary much under different circumstances, and depend especially on whether they have been in the atmosphere or not. Consequently, in the experiments described in the following pages, care was taken that the films were deposited at a low pressure in the tube in which measurements were to be made, and immediately after depositing, as high a vacuum as possible was obtained, and maintained during the course of some of the experiments. Measurements were made of the photoelectric velocities and currents for incident and emergent light.

\section{Description of the Apparatus.}

The quartz plate $A\left(1 \frac{1}{2} \mathrm{~mm}\right.$. thick) was attached to a thick brass rod B, which was fixed by sealing-wax through

Fig. 1.

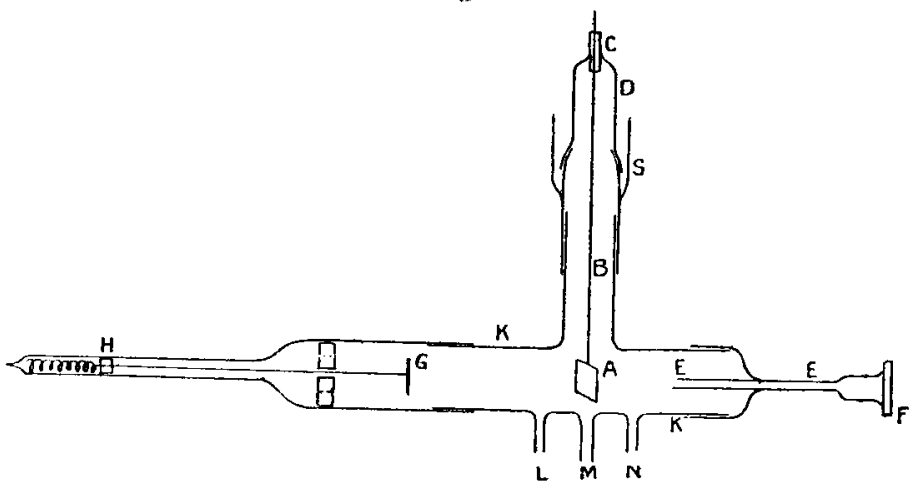

an amber plug $\mathrm{C}$, which in its turn was fixed into one part $D$ of a ground joint $S$. By means of this ground joint the quartz plate $\mathrm{A}$ could be made to take up any angle with regard to the direction of the light through the tube EE. This tube was narrow so as to admit only a thin beam of light. The quartz plate $F$ closed the tube $E$. G was a platinum electrode attached rigidly to a rod, at the other end of which was au iron ring $H$. By applying an electromagnet from outside to this iron ring, the system $\mathrm{HG}$ could be moved along the tube KK. Before the quartz plate A and the platinum electrode $G$ were inserted, the walls of the tube KK were coated thickly with platinum by stretching a platinum wire through the tube, and passing a current between it as cathode, and a wire in one of the side tubes 
LMN at a low pressure. A wire passing through the tube $\mathrm{L}$ was made to touch the walls so that the potential of the latter could be controlled. The tube $M$ was used for connecting to the mercury-pump, and through $\mathrm{N}$ a wire protruded a short distance into the tube $K$. This wire was used as anode when the current was passed from the platinum electrode $G$ as cathode to deposit a film on the quartz plate $A$.

Before this was done, $G$ was kept as far as possible away from $A$, and the current passed for a long time to drive out some of the occluded gas. Then $G$ was moved up to about $1 \mathrm{~cm}$. from $A$ and the film deposited.

The rod B was connected in the ordinary way to one pair of quadrants of a Dolezalek electrometer. In making measurements of the velocities of the electrons, all stray wires, $L, M$, and the platinum electrode $G$ were earthed, so that the thin film was in an earth-connected cylinder. The maximum potential taken up by the film nnder the action of ultraviolet light was measured, when it was turned to face the light (incident), and when it was turned away from the light (emergent). The velocity $v$ of an electron is connected with this maximum potential $\vec{V}$ by the formula $\frac{1}{2} m v^{2}=e V$.

Scales were pasted around the two portions of the ground joint so as to show exactly what position the quartz plate occupied. A quartz mertury lamp was used as source of light.

The measurements of the photoelectric current were made by shunting the pairs of quadrants of the electrometer by a high resistance. This resistance was made by depositing platinum on glass. It was found that if the vessel in which this was done was pumped out as far as possible and sealed off, a very constant high resistance. was obtained, which is very suitable for the present purpose. The currents were measured for different fields between the film and the walls of the vessel, which fields were obtained by charging the walls to different potentials.

The relative thicknesses of the films are given in terms of the lengths of time taken to deposit them.

The last column gives the ratio of the emergent velocity to the incident velocity. It will be noticed that for the films 40 minutes and 50 minntes, different values are given for the actual velocities. These different values for the same film were obtained under different conditions. The measurements were always made at the highest vacuum it was possible to obtain, but on a few occasions it was necessary to let air into the tube. On pumping out again, it was 


\section{Table $I$.}

Measurement of the velocities of the electrons produced by incident and emergent lights.

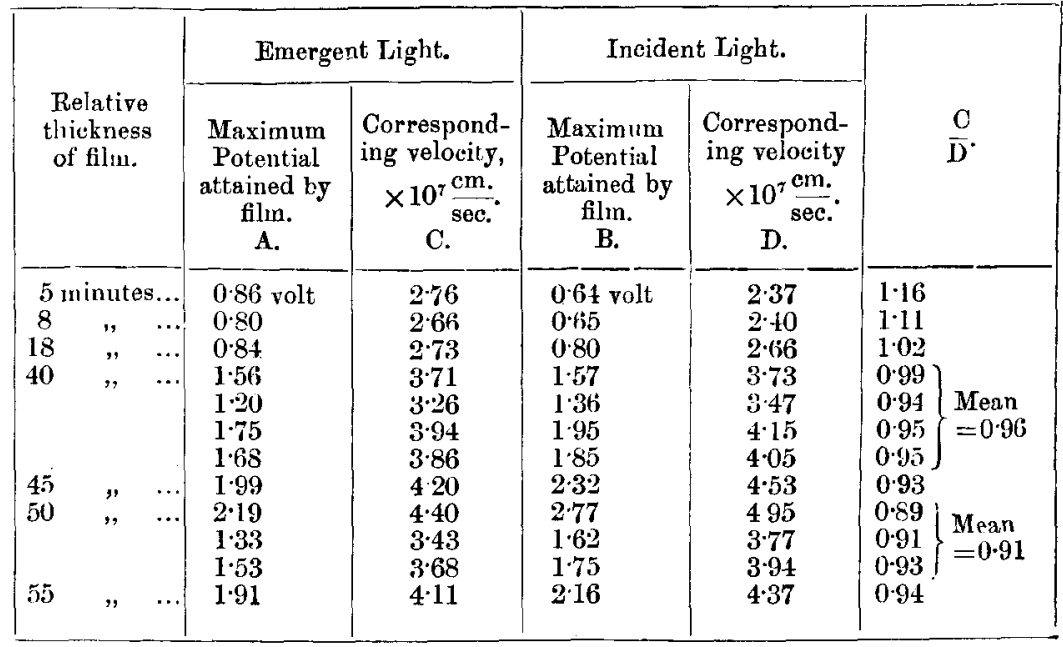

Fig. 2 shows gruphically the relation between $\frac{\mathrm{C}}{\mathrm{D}}$ and the thickness of the film.

Fig. 2.

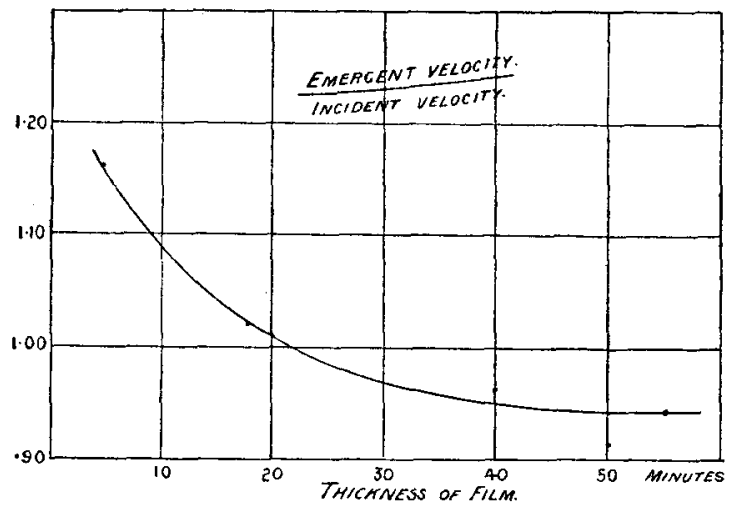

found that the actual velocities had diminished both for incident and emergent light. On standing in a vacuum for some time the velocities increased again, but not to exactly the same values as before letting in air. It will be noticed that the ratio of the emergent to incident velocities has not varied much, although the actual velocities varied.

Phil. Mag. S. 6. Vol. 23. No. 136. April 1912. 20 
This seems to show that occluded gas in the film influences the actual magnitudes of the velocities, though not the ratio for the incident to emergent effects. The values given for the velocities represent steady values which are only obtained some time after the film was deposited. Immediately after a film has been deposited the velocities vary considerably, but more for incident than for energent ligbt. A constant ratio was only obtained some time after depositing. Frequent attempts were made to find whether it was possible to eliminate these initial variations by passing the current for a long time before depositing the film, so as to get rid of occluded gas from the platinum electrode, but so far it has not been found possible to do this.

Fig. 3.-Film of 5 minutes.

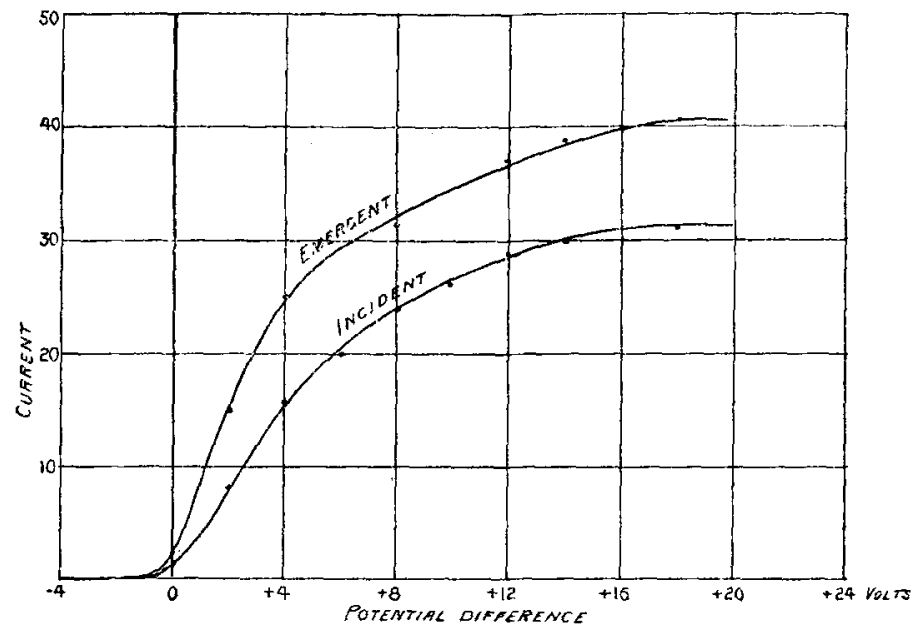

Fig. 4.-Film of 8 minutes.

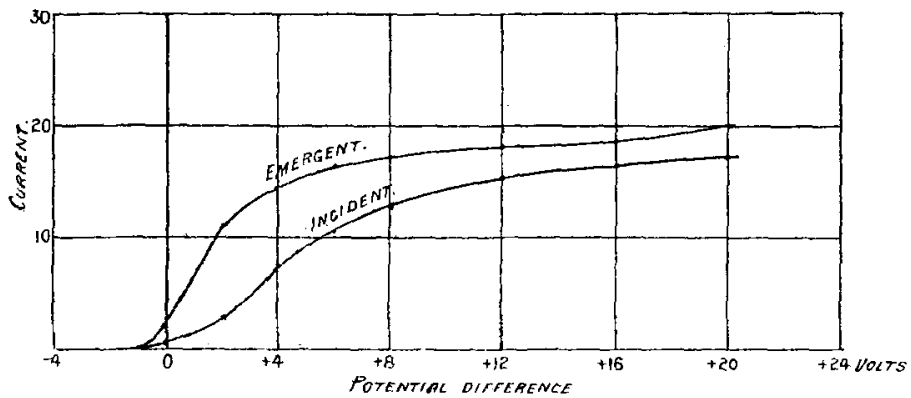

The results of Table II. are shown graphically in figs. 3-6. It is seen that in every case the photoelectric current 
Properties of Thin Hetal Films.

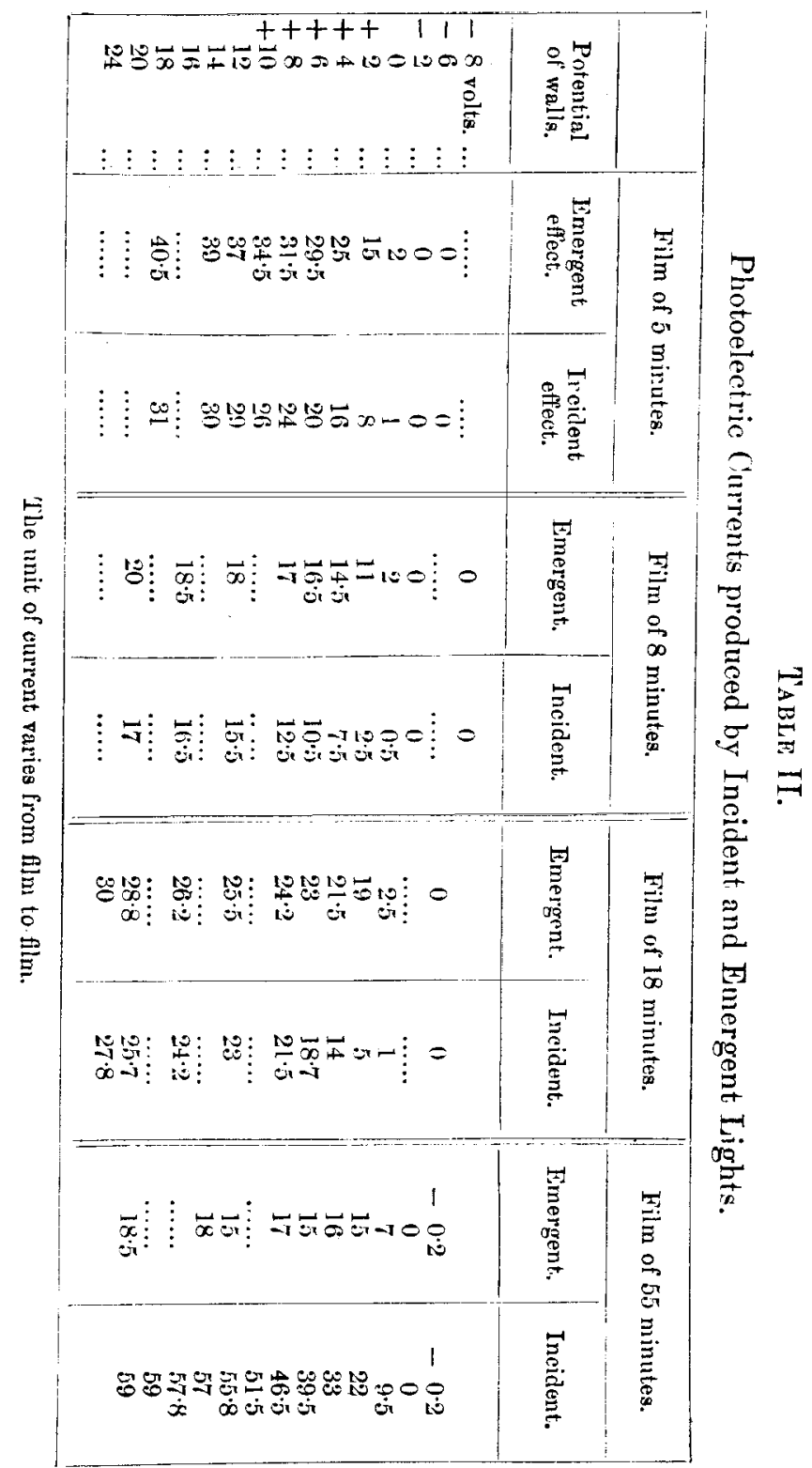


approaches a maximum value as the potential difference between the film and the walls of the vessel increases, so as to drive electrons away from the film. Also in all but one case it is noticed that there is no inverse photoelectric effect,

Fig. 5.-Film of 18 minutes.

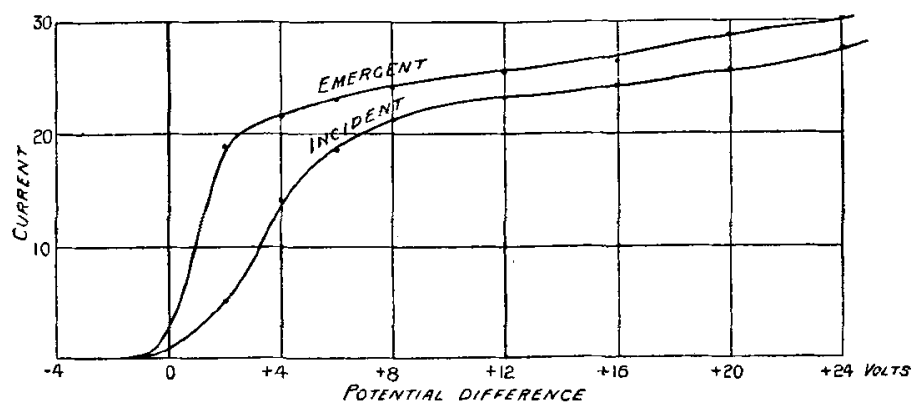

Fig. 6.-Film of 55 minutes.

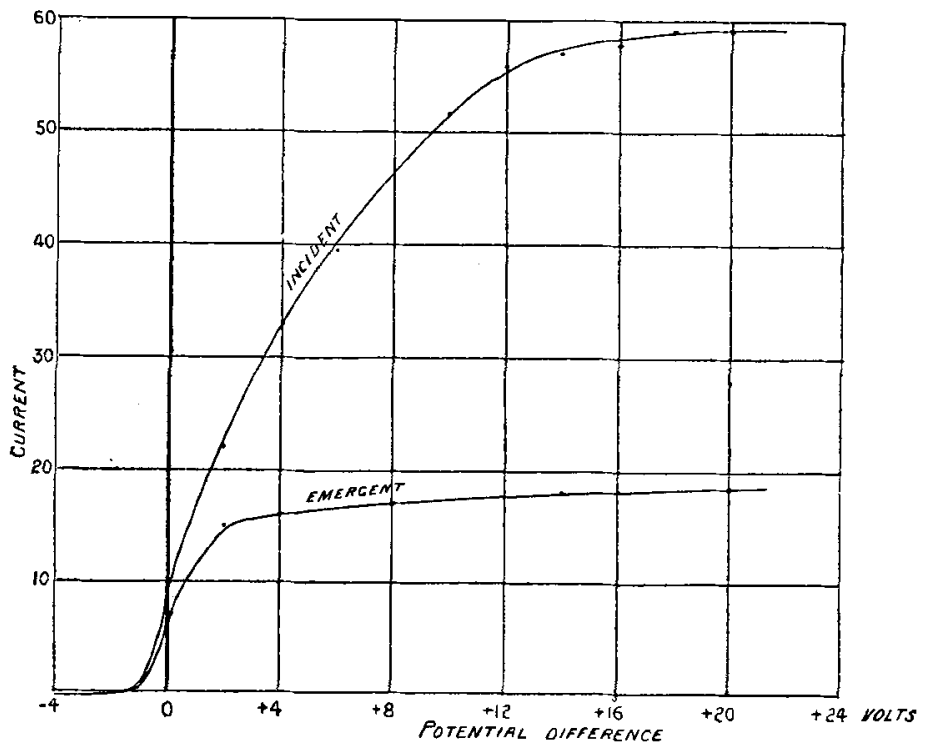

which shows that the influence of reflected light can be neglected. Here, again, as in the case of the velocities, an influence of the thickness of the film is noticeable. For thin films the maximum photoelectric current is larger for emergent than for incident light, and for thick films the reverse is 
the case. In the following table the ratio of the maximum currents is shown in relation to the thickness of the film.

TABLE III.

\begin{tabular}{|c|c|c|c|c|c|}
\hline \multirow{2}{*}{\multicolumn{3}{|c|}{$\begin{array}{c}\text { Relative thickness } \\
\text { of film. }\end{array}$}} & \multicolumn{2}{|c|}{ Maximum current. } & \multirow{2}{*}{$\frac{\mathrm{A}}{\overline{\mathrm{B}}}$. } \\
\hline & & & $\begin{array}{c}\text { Emergent. } \\
\text { A. }\end{array}$ & $\begin{array}{c}\text { Incident. } \\
B .\end{array}$ & \\
\hline \multicolumn{3}{|c|}{5 minutes ... } & 40 & 31 & 1.29 \\
\hline 8 & , & & 18 & 16 & $1 \cdot 12$ \\
\hline 18 & , & & 30 & 27 & $1 \cdot 11$ \\
\hline 55 & : & ......... & 18 & 59 & $0 \cdot 31$ \\
\hline
\end{tabular}

Discussion of Resulls.

Velocities.-Fig. 2 shows that for thin films the velocities of the electrons for emergent light are greater than those for incident light, and that for thick films the reverse is the case. The velocities measured were the maximum velocities in each case, which, according to Ladenburg *, refer to the shortest wave-lengths of light used. Thus one particular wave-length of light produces quicker electrons for emergent than for incident light, when thin films are used, and vice versa for thick films.

It was thought that possibly the results for thick films might be due to some selective absorption of light by them. Thus if the shortest wave-lengths are absorbed conpletely before the light has penetrated far into the film, then the velocities of the emergent electrons will be smaller than those of the incident. It was not found possible to substantiate this possibility by taking photographs of the spectrum of the lamp used. A Hilger quartz spectroscope was used and photographs taken :-

1st. With nothing between the lamp and the spectroscope.

2nd. With a film of 18 minutes deposit before the slit.

$$
\text { 3rd. " " } " 55 \quad "
$$

In each case a number of exposures of different lengths of time were made, and although a general absorption of light by the films was observed, yet it was not possible to detect any selective absorption. The shortest wave-lengths 
could be photographed through the thickest film used. The wave-length of this line was 2302 . The spectrum was a line spectrum.

Currents.-The results obtained from the measurements of currents are :-

1. That for thin films the maximum emergent current is larger than the maximum incident current, and vice versa for thick films.

2. For emergent light, the current rises rapidly with potential difference at first, and approaches its maximum value for a small value of this potential. The turning-point of the curves between the rapid increase and the slow increase to the maximum value, is at about 2 volts for the 18 -minute and the 55 -minute films, and at about 4 volts for the 8-minute film. For incident light, the rise of the current with the potential difference is not so great at first as for emergent light, and the turning-points to the saturationvalues are at much higher potentials, of the order $8-12$ volts.

These results show that light assists electrons to leave a metal more in the direction of the light than in the opposite direction for thin films, but when the films are thrick the reverse is the case.

Without attempting to explain the whole phenomena, attention might be cailed to the difference in the form of the curves for incident and emergent currents (figs. 3-6). This difference cannot, I think, be explained by a dissymmetry in the tube.

An explanation of the form of the curve for thick metals was put forward by Ladenburg and Markau *. They supposed that the same number of electrons leave an electrode no matter what the potential difference between it and the receiving electrode is. The fact that a certain potential difference is necessary in order to obtain the maximum current, they explain as being due to electrons being reflected from the receiving electrode and getting back to the emitting electrode when the potential difference is small. If this is the case, then the form of the curve ought to be the same for incident and emergent light. Again, in the present experiments the receiving electrode is the walls of the vessel, and so is perpendicular to the emitting electrode, so that any reflected electrons ought to go to other parts of the walls and not get back to the film.

The original explanation of the form of the curve given by Lenard $\dagger$, that a certain potential difference is necessary

* Verh. d. deutsch. Phys. Ges. 1908, n. 14, p. 562.

† Amn. der Phys. riii. 1902, p. 149. 
to drive the electrons away from the surface, is more in harmony with the present experiments. According to this view a larger potential difference is necessary to drive electrons away from a film for incident light than for emergent light, thus showing that the direction of the light has something to do with the emission of electrons.

Approximate values of the actual thicknesses of the films used can be calculated in the following way :-

Drude's values * for the absorption coefficient and reflexion coefficient for platinum for sodium light is $45,000 \mathrm{~mm}^{-1}$ and $\cdot 70$ respectively. It is doubtful whether the same values hold for thin films, but an approximation can be made to the true state of affairs by assuming these values for the whole spectrum and for thin films. The thickest film used ( 55 minutes) let through about 20 per cent. of the light which fell on it. This was measured photometrically and also estimated from the spectra. 30 per cent. of the light falling on the film enters it, and thus 10 per cent. is absorbed. This means that a film of 55 minutes deposit absorbs $\frac{1}{3}$ of the light which enters it. The intensity of light falls off according to an exponential law. On plotting the curve $y=e^{-45,300 x}$ it is found that an absorption of $33 \cdot 3$ per cent. corresponds to a thickness of $10^{-6} \mathrm{~cm}$.

The thicknesses of the other films can be readily calculated once the thickness of one has been determined.

I have great pleasure in recording my best thanks to Prof. Hicks and Dr. S. R. Milner for the interest they have taken in this work, and for much valuable advice in connexion with it.

University of Sheffield, Dec. 6, 1911.

XLIX. The Virial of a Mixture of Ions. By S. R. Milner, D.Sc., Lecturer in Physics, The University, Sheffield $†$.

TWHE problem considered in this paper is the determination of the average virial of a mixture of $\mathrm{N}+$ and $\mathrm{N}$ - ions contained in a volume $\mathrm{V}$, on the assumption that every + ion repels every other + ion, and attracts every - ion, with a force $q^{2} / r^{2}$, where $r$ is the distance between the pair of ions considered, and $q$ the ionic charge. The following statement defines both the average virial and also the strict theoretical method of calculating it:-For a

* Wied. Ann. 1890, Bd. xxxix. p. 481.

+ Communicated by the Author. 